\title{
Malignant mesothelioma in situ: morphologic features and clinical outcome
}

\author{
Andrew Churg ${ }^{1} \cdot$ Francoise Galateau-Salle ${ }^{2} \cdot$ Anja C. Roden $^{3} \cdot$ Richard Attanoos $^{4} \cdot$ Jan H. von der Thusen $\mathbb{1 0}^{5}$. \\ Ming-Sound Tsao ${ }^{6} \cdot$ Nina Chang ${ }^{6} \cdot$ Marc De Perrot $^{7} \cdot$ Sanja Dacic ${ }^{8}$
}

Received: 11 July 2019 / Revised: 23 July 2019 / Accepted: 23 July 2019 / Published online: 2 August 2019

(c) United States \& Canadian Academy of Pathology 2019

\begin{abstract}
The existence of an in situ phase of malignant mesothelioma has long been postulated but until recently has been impossible to prove. Here we describe ten patients with mesothelioma in situ, defined by a single layer of surface mesothelial cells showing loss of BAP1 nuclear immunostaining, no evidence of tumor by imaging and/or by direct examination of the pleura/ peritoneum, and no invasive mesothelioma developing for at least 1 year. Nine cases were pleural and one peritoneal. Most patients were biopsied for repeated effusions of unknown etiology; in two patients mesothelioma in situ was found incidentally in lung cancer resections. In addition to surface mesothelium with BAP1 loss, one case had a surface papillary proliferation with BAP1 loss, and two cases had a small (few millimeter) nodule with BAP1 loss. CDKN2A was deleted by FISH in one of eight cases. Methylthioadenosine phosphorylase showed partial loss in the surface mesothelium by immunohistochemistry in three cases. Invasive malignant mesothelioma developed in seven patients with time between biopsy and invasive disease from 12 to 92 (median 60) months. Invasive mesothelioma has not developed in the other three patients at 12, 57, and 120 months, but the latter patient, who has pleural plaques, still has repeated pleural effusions, probably representing a so-called "benign asbestos effusion." We conclude that mesothelioma in situ, as diagnosed using the criteria outlined above, is associated with a high risk of developing invasive mesothelioma, but typically over a relatively protracted time, so that curable interventions maybe possible.
\end{abstract}

Andrew Churg

achurg@mail.ubc.ca

1 Department of Pathology, Vancouver General Hospital and University of British Columbia, Vancouver, BC, Canada

2 Centre National Référent MESOPATH, Centre Leon Berard, Lyon, France

3 Department of Laboratory Medicine and Pathology, Mayo Clinic, Rochester, MN, USA

4 Department of Cellular Pathology, University Hospital of Wales, and School of Medicine, Cardiff University, Cardiff, Wales, UK

5 Department of Pathology, University Medical Center, Rotterdam, The Netherlands

6 Department of Pathology, University Health Network and Department of Laboratory Medicine and Pathobiology, University of Toronto, Toronto, ON, Canada

7 Division of Thoracic Surgery, University Health Network, University of Toronto, Toronto, ON, Canada

8 Department of Pathology, University of Pittsburgh Medical Center, Pittsburgh, PA, USA

\section{Introduction}

Malignant pleural mesothelioma is an invariably fatal disease while malignant mesothelioma of the peritoneum requires a major surgical procedure in the form of tumor debulking followed by hot intraperitoneal chemotherapy which maybe combined with systemic chemotherapy. Although patients treated in this fashion may have prolonged survival, it is unclear whether any are actually cured.

Malignant tumors in many organs have an in situ phase that can be identified morphologically, and when detected allows ablation or excision of the affected region to prevent the development of invasive disease. By analogy with other organs, malignant mesothelioma should have an early in situ phase that, if found and destroyed, could offer a chance of cure. However, the pathology literature on mesothelioma in situ is controversial: a few reports $[1,2]$ have described what the authors believed was mesothelioma in situ, but all in cases with invasive mesothelioma as well, so that the putative in situ disease may well have been spread of the invasive tumor along the pleural surface. A 
further problem is that reactive mesothelial cells can be cytologically very atypical. The current consensus is that there is no reliable method, based on routine morphology, to separate mesothelioma in situ from reactive surface mesothelial proliferation, and for that reason there is a general recommendation against making a diagnosis of mesothelioma in situ [3-5].

BAP1 (BRCA-1 associated protein-1) is a gene with a complex set of functions, including acting as a nuclear deubiquitinase, a controller of DNA repair and cell proliferation, and also as a cytoplasmic mediator of mitochondrial calcium flux and apoptosis. There is an increasing belief that BAPl is a tumor suppressor gene [6]. Somatic mutations in BAPl are found in around $60 \%$ of malignant mesotheliomas, and biallelic loss or mutation can be detected by loss of normal nuclear staining using immunohistochemistry. A recent meta-analysis of over 1800 published mesothelial biopsy and cytology cases concluded that nuclear BAP1 loss as detected by immunohistochemistry is $100 \%$ specific for malignant mesothelioma vs reactive mesothelial hyperplasia [7].

We previously [8] utilized this information to report two cases of what we concluded were true malignant mesothelioma in situ, characterized by a surface proliferation of mesothelial cells that showed BAP1 loss on immunohistochemistry. One of these cases had very focal and very shallow invasion into fat, but the other case only surface mesothelium with loss of BAP1 and no invasion; that patient has now developed invasive mesothelioma (Case 1 in this report). To further understand the morphologic features and the potential of mesothelioma in situ for development of invasive disease, we have collected ten cases using the following definition: (1) a surface proliferation of mesothelial cells in the form of a single layer of mesothelial cells that had lost BAP1; (2) no evidence of invasive tumor by imaging and/or direct visual inspection of the pleura or peritoneum at the time of biopsy; (3) no invasive mesothelioma diagnosed for at least 1 year after the biopsy. The latter criterion is intended to avoid calling a biopsy mesothelioma in situ that is actually a suboptimal sample of a concurrent invasive mesothelioma that has spread along the serosal surface. Here we report our findings with these cases.

\section{Material and methods}

This study was approved by the Committee on Human Ethics of the University of British Columbia. Pathologists with an interest in malignant mesothelioma were contacted to see if they had cases that matched the criteria laid out in the "Introduction" section. Where tissue was available, immunohistochemical staining for methylthioadenosine phosphorylase (MTAP) and FISH for detection of loss of
$C D K N 2 A$ were run. Presenting clinical information and follow-up data were also collected.

BAP1 immunostaining was performed in each originating laboratory using a variety of protocols, but all laboratories employed mouse monoclonal antihuman BAP1 clone C4 (Santa Cruz Biotechnology Inc., Dallas, TX). MTAP staining was performed as described [9] on a DAKO OMNIS machine using antibody clone 2G4 (Abnova, Walnut Creek, CA) at a primary antibody dilution of 1:100 after heat-induced epitope retrieval at $\mathrm{pH} 9.0$ for $40 \mathrm{~min}$. Blocking was carried out with Dako Envision Flex Peroxidase-Blocking reagent, and visualization with Dako Envision Flex HRP reagent. For MTAP loss of cytoplasmic staining was viewed as loss and nuclear staining was ignored [9]; for BAP1 only loss of nuclear staining was viewed as loss. Positive staining of inflammatory or stromal cells was required as an internal control for interpretation of BAP1 and MTAP stains.

Dual-color FISH was performed for $C D K N 2 A$ as previously described [10]. CDKN2A was assessed using a Spectrum-Orange-labeled, locus-specific probe (Abbott Molecular, Des Plains, IL, USA) as well as SpectrumGreen-labeled chromosome 9 centromeric (CEP9) probe. At least 60 cells were scored for each case and control. Each tumor was assessed by the average and the maximum numbers of copies of the either $C D K N 2 A$ per cell or the average ratio of the gene to CEP9 copy numbers. A case was considered to be positive for deletion if homozygous deletion was identified in at least $20 \%$ of nuclei.

\section{Results}

Table 1 shows the demographic data for the study cases: there were six men and four women with an average age of 69 and a range of 53-79. Nine patients had pleural disease, of which six had repeated pleural effusions of unknown etiology and one (case 8) had repeated pleural effusions that probably represented so-called benign asbestos effusions. One patient presented with ascites. In two cases there were no effusion but the patients had undergone resection for a primary lung carcinoma and mesothelioma in situ was an incidental finding on microscopic examination. No patients had radiologic evidence of tumor, although smooth pleural thickening was found in two; where the pleural or peritoneal surface was examined visually there was also no evidence of tumor.

By definition all cases had a single layer of surface mesothelial cells that showed BAP1 loss by immunohistochemistry. These cells were sometimes flat but more often cuboidal and had various degrees of cytologic atypia; however, in most cases atypia was nonexistent to minimal (Figs. 1, 2). In one of the lung cancer resection specimens (case 7) a small papillary pleural proliferation that resembled 


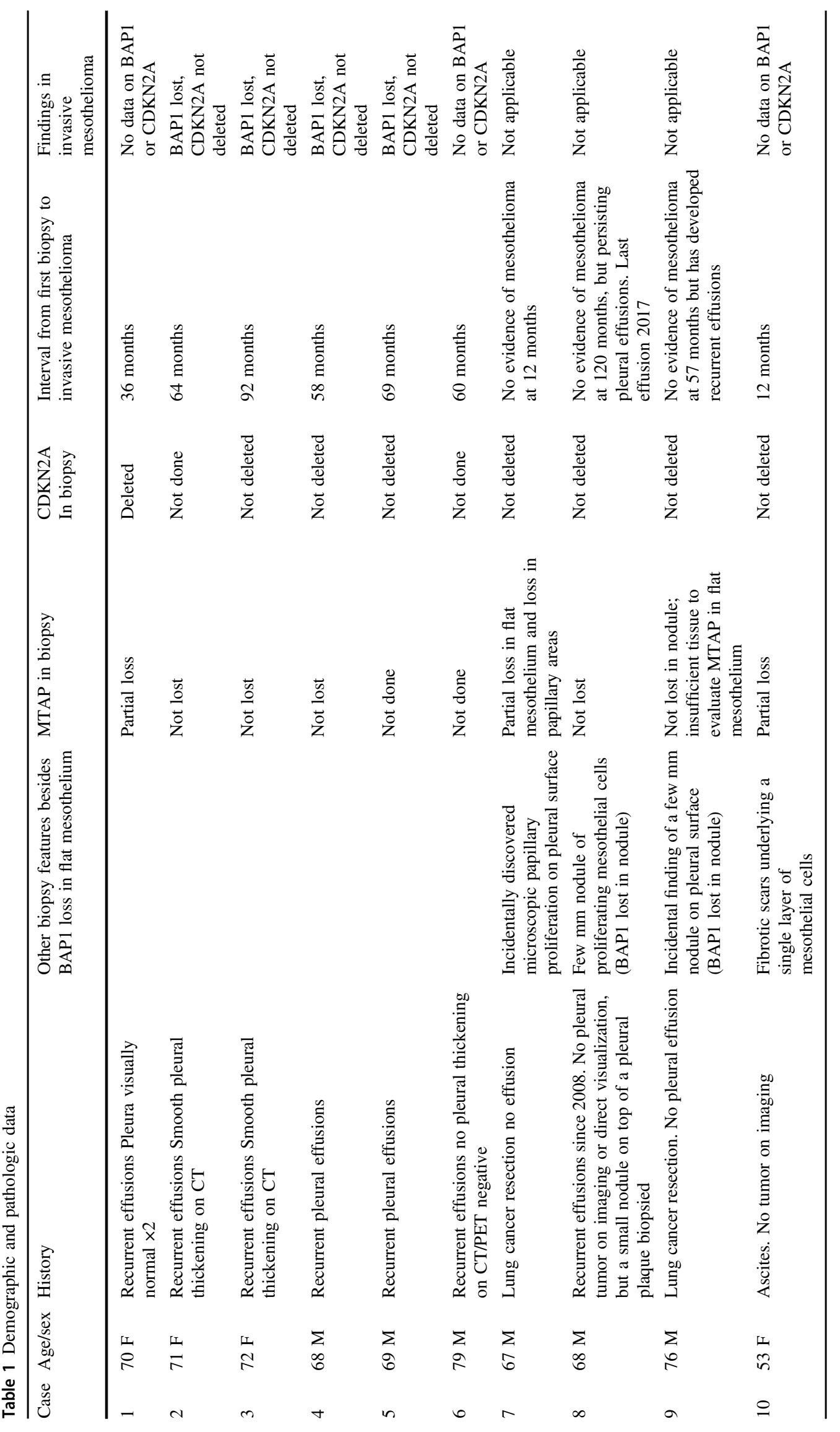




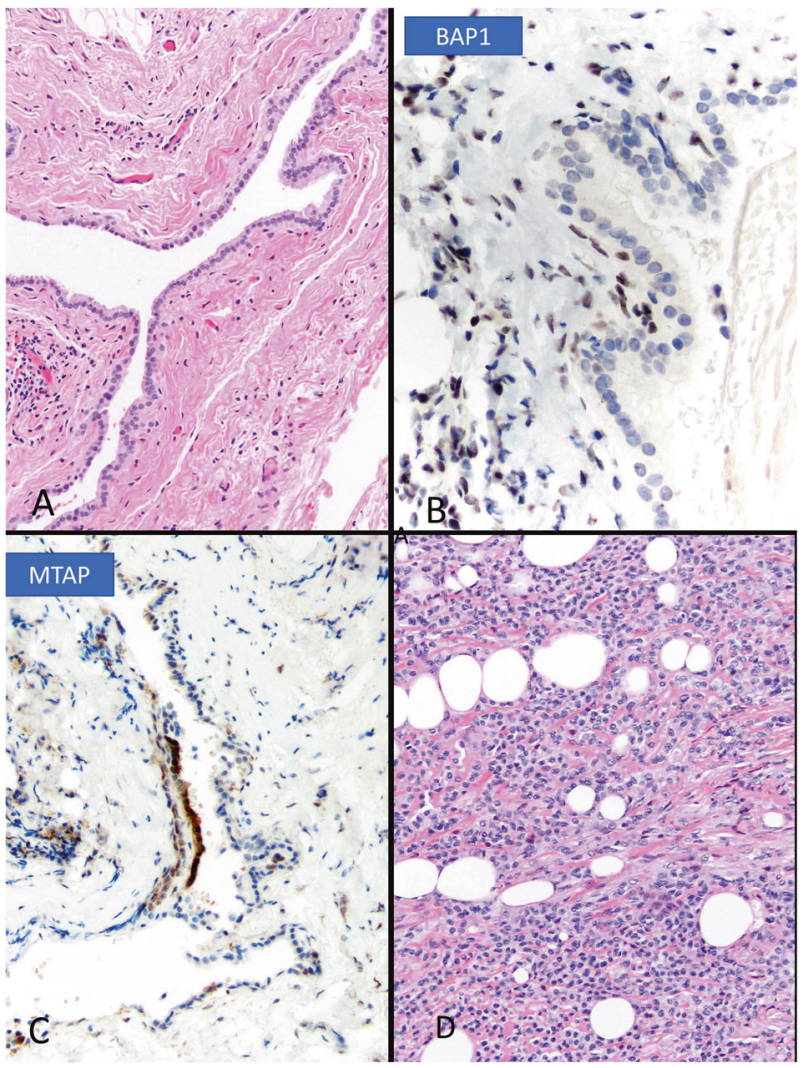

Fig. 1 (Case 1): a Typical appearance of mesothelioma in situ as a single layer of innocuous-appearing cuboidal mesothelial cells. b Loss of BAP1 in the flat mesothelium. c MTAP staining shows a mixture of apparent loss with areas of retained staining. d Invasive mesothelioma that developed at 36 months

a well differentiated papillary mesothelioma was found incidentally (Fig. 2); however, as opposed to well differentiated papillary mesothelioma, the mesothelial cells in the papillary area demonstrated quite marked cytologic atypia (Fig. 2). After the papillary proliferation was found to have loss of BAP1, an additional block of flat mesothelium was stained and also showed loss of BAP1. The other case that was a lung cancer resection specimen had an incidentally detected few millimeter pleural nodule of proliferating mesothelial cells (Fig. 3) that had lost BAP1 (the nodular proliferation was reported as case 19 in reference [11] and is case 9 here); again, staining of an additional block showed flat mesothelium that had lost BAP1 (Fig. 3). In one case (case 8) biopsy had shown a small nodule of proliferating mesothelial cells that lacked BAP1 and retrospective review of the biopsy showed an area of flat mesothelium that had also lost BAP1.

MTAP immunohistochemistry was run on the flat mesothelium in seven cases. Four cases did not show any loss and three showed patchy loss (Figs. 1,2) in the flat mesothelium. MTAP was not run or there was insufficient tissue in three cases. $C D K N 2 A$ FISH showed homozygous deletion in only one of eight cases (case 1).
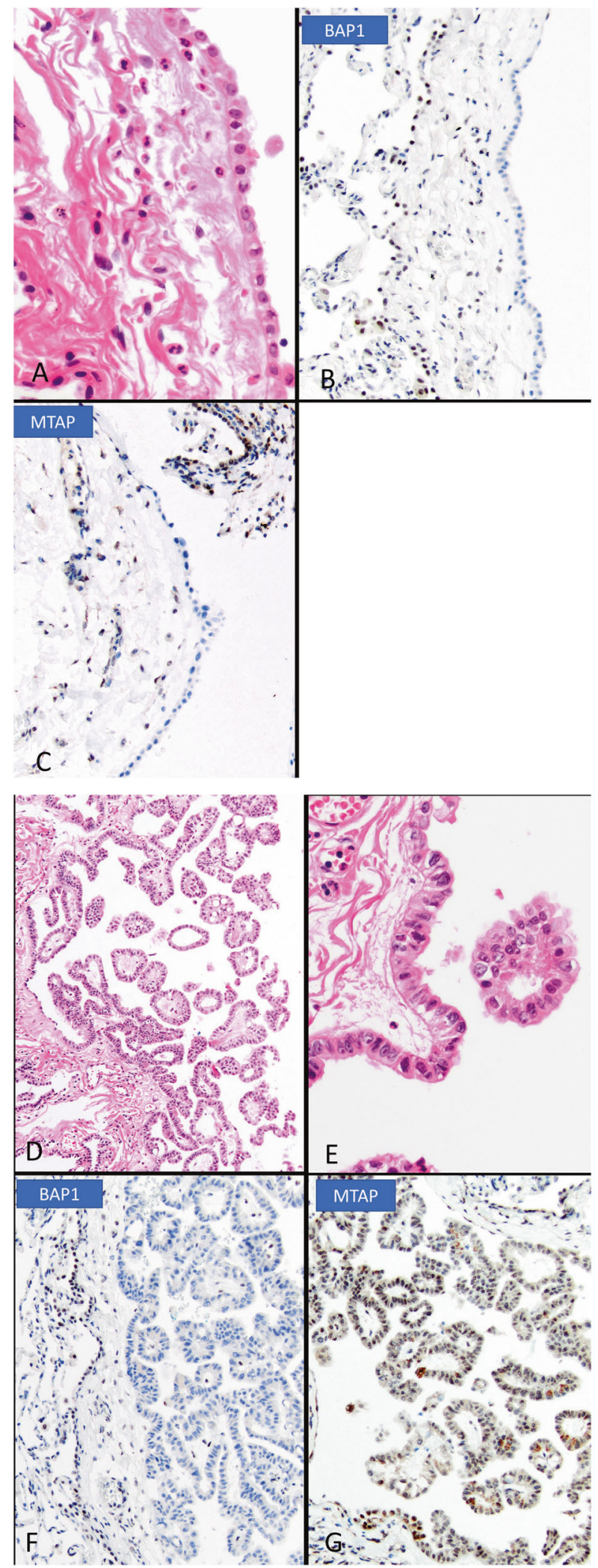

Fig. 2 (Case 7): a Single layer of cuboidal mesothelial cells, in this case with some degree of cytologic atypia. b Loss of BAP1 in the surface mesothelial cells. c MTAP staining shows partial loss. d, e Low and high power views of a papillary area on the pleural surface with marked cytologic atypia. f BAP1 is lost in the papillary area. g Cytoplasmic MTAP staining is lost in the papillary area 


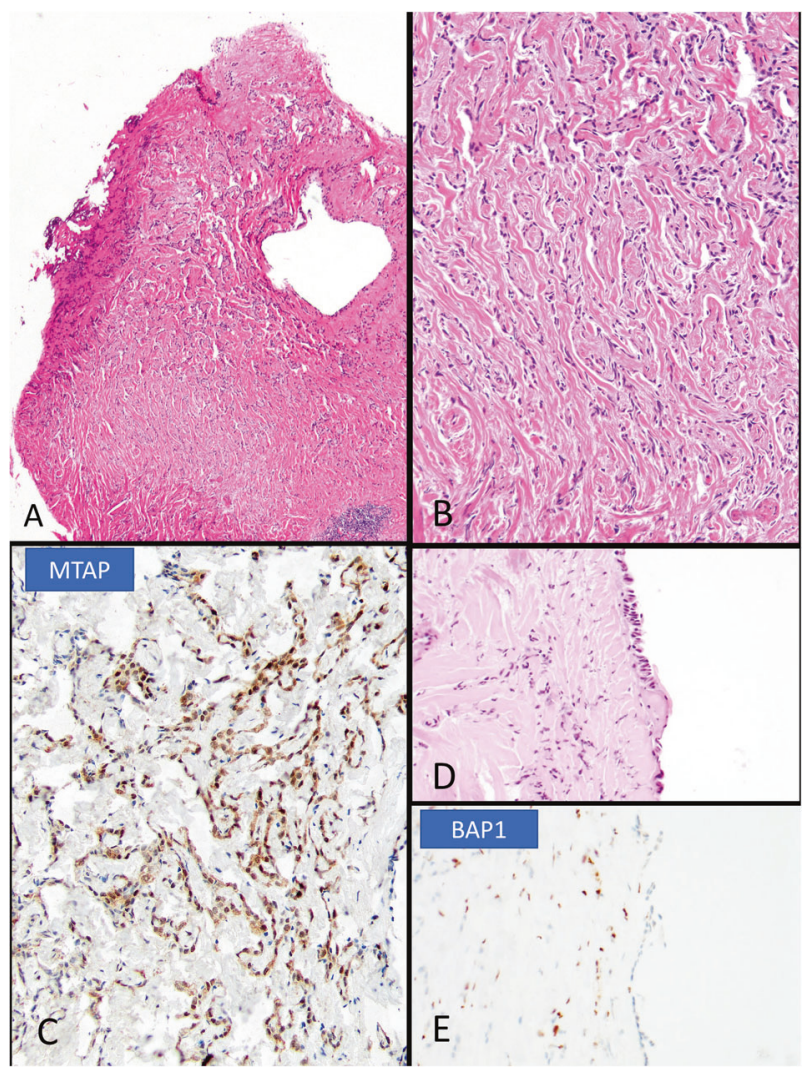

Fig. 3 (Case 9): a lung cancer resection specimen with a few millimeter nodule of proliferating mesothelial cells $(\mathbf{a}, \mathbf{b})$ that have lost BAP1 but not MTAP (c). Although this appearance mimics an invasive mesothelioma, the remainder of the specimen showed only flat mesothelium with BAP1 loss (d, e). Patient is alive at 57 months without overt mesothelioma but has developed recurring pleural effusions

Follow-up data showed development of an invasive malignant mesothelioma in seven patients (six pleural, one peritoneal) with a time interval from biopsy to invasive tumor of 12-92 months (median 60 months). As of the time of writing, three patients had not developed invasive disease with time intervals of 12, 57, and 120 months. One of these patients (case 9) has developed recurrent effusions but has no overt tumor at 57 months, and another (case 8) has persisting pleural effusions and pleural plaques but also no overt tumor at 120 months (see "Discussion" section). Data on BAP1 and CDKN2A deletion was available for four of the invasive tumors; all showed BAP1 loss and none showed $C D K N 2 A$ deletion.

\section{Discussion}

The most striking finding to emerge from this study is that BAP1 loss in flat surface mesothelium confers a very high risk of subsequent appearance of invasive tumor. In this series $70 \%$ of such patients developed an invasive mesothelioma. This figure is remarkable in comparison to carcinoma in situ at other sites; for example, Ostor [12] reported that $33 \%$ of cervical
CIN3 lesions regressed, while only $12 \%$ progressed to invasive squamous carcinoma. The other notable finding from our data is that progression to invasive mesothelioma is usually not a very rapid process. The median time to progression here was 60 months, although this number maybe biased upwards because we would not have accepted a case with a shorter time to progression for inclusion in this series, in order to avoid the risk of misinterpreting a nonrepresentative sample of an invasive mesothelioma.

A second important conclusion is that routine morphology will generally not detect mesothelioma in situ because the mesothelial cells of mesothelioma in situ tend to be very bland, and there is no visual clue to their true nature (vs reactive mesothelial cells). Only one case in this series demonstrated significant cytologic atypia in the mesothelium (case 7, Fig. 2). Thus clinical information is vital: in a patient with a story of recurrent effusions and no radiologic or visual evidence of tumor, BAP1 staining is crucial to making the correct diagnosis.

Deletion of $C D K N 2 A$ is a common finding in malignant mesotheliomas. MTAP is located close to $C D K N 2 A$ at $9 \mathrm{p} .21$ and is frequently co-deleted with $C D K N 2 A$ [13]. MTAP immunohistochemistry can serve as a surrogate for $C D K N 2 \mathrm{~A}$ FISH in invasive mesotheliomas because most cases with $C D K N 2 A$ homozygous deletion also show complete loss of MTAP immunohistochemical staining $[9,14,15]$. With the in situ lesions described here, however, MTAP loss did not consistently correlate with CDKN2A FISH loss. This may imply that in these in situ lesions MTAP is hypermethylated rather than mutated or deleted, although MTAP hypermethylation was not seen in a recent report on invasive malignant mesotheliomas [16]. It is conceivable that these findings reflect-evolving clones with MTAP loss. It is also surprising that only one of eight examined cases had $C D K N 2 A$ deletion, which is much lower than what has been reported in an invasive malignant mesothelioma. Large scale molecular studies in malignant mesotheliomas have shown that $B A P 1$ mutations and $C D K N 2 A$ deletions are not mutually exclusive events $[17,18]$. Therefore, we can hypothesize that BAPl mutation is an early event in mesothelial carcinogenesis and $M T A P / C D K N 2 A$ deletion a later event. Unfortunately, because most of these cases were referred as consultation cases to the authors of this study, we were not able to obtain tissue to perform a comprehensive molecular analysis of mesothelioma in situ and matched invasive mesothelioma that would better elucidate the sequence of events in the progression and development of invasive mesothelioma.

Three cases in this series did not progress to overt invasive disease. One of them (case 9) had a small (few millimeter) nodule that was incidental finding in a lung cancer resection specimen; this patient is alive but has developed persisting 
effusions at 57 months. Because there was also flat mesothelium with loss of BAP1, the case clearly has mesothelioma in situ and we have included it for that reason. One could argue that this nodule really represents an early tiny clone of invasive mesothelioma, but there is no fat or lung invasion, and the nodule is very small and is solitary, so that a clear diagnosis of invasive mesothelioma is not possible on this material. What is really needed at this point is a molecular marker(s) that separates invasive from in situ disease.

One of the other cases (case 8) is noteworthy because the patient also had a small nodule, but this patient has had 10 years of recurrent effusions and has still not developed invasive disease. This patient has pleural plaques and the clinical process fits best for benign asbestos effusion (clearly a misnomer in this instance). In situ disease can also be papillary as seen in case 7 and Fig. 2 .

One conclusion that is implied in our data is that recurrent unilateral effusions with no obvious etiology should be viewed with considerable suspicion and the pleura/peritoneum biopsied, even if there is nothing radiologically to suggest a malignancy and the pleura/peritoneum is visually normal. Given the risk of invasive mesothelioma, an even stronger case can be made for performing a pleural biopsy in patients with what clinically appear to be benign asbestos effusions, such as case 8 . By the same token, the pathologist should perform BAP1 staining on any case with a history of recurrent effusions, no matter how bland the surface mesothelium appears. Lastly, although loss of BAP1 in surface mesothelium carries an ominous long-term prognosis, the relatively extended time to development of invasive mesothelioma potentially allows a therapeutic intervention before that event occurs.

\section{Compliance with ethical standards}

Conflict of interest The authors declare that they have no conflict of interest.

Publisher's note: Springer Nature remains neutral with regard to jurisdictional claims in published maps and institutional affiliations.

\section{References}

1. Whitaker D, Henderson DW, Shilkin KB. The concept of mesothelioma in situ: implications for diagnosis and histogenesis. Semin Diagn Pathol. 1992;9:151-61.

2. Bono F, Lombardi M. Prominent in situ component in a pleural malignant mesothelioma. Int J Surg Pathol. 2015;23:127-9.
3. Henderson DW, Shilkin KB, Whitaker D. Reactive mesothelial hyperplasia vs mesothelioma, including mesothelioma in situ: a brief review. Am J Clin Pathol. 1998;110:397-404.

4. Churg A, Galateau-Salle F. The separation of benign and malignant mesothelial proliferations. Arch Pathol Lab Med. 2012;136:1217-26.

5. Henderson DW, Reid G, Kao SC, van Zandwijk N, Klebe S. Challenges and controversies in the diagnosis of mesothelioma: part 1. Cytology-only diagnosis, biopsies, immunohistochemistry, discrimination between mesothelioma and reactive mesothelial hyperplasia, and biomarkers. J Clin Pathol. 2013;66:847-53.

6. Bononi A, Giorgi C, Patergnani S, Larson D, Verbruggen K, Tanji $\mathrm{M}$, et al. BAP1 regulates IP3R3-mediated $\mathrm{Ca}^{(2+)}$ flux to mitochondria suppressing cell transformation. Nature. 2017;546:549-53.

7. Wang LM, Shi ZW, Wang JL, Ly Z, Du FB, Yang QB, et al. Diagnostic accuracy of BRCA1-associated protein 1 in malignant mesothelioma: a meta-analysis. Oncotarget. 2017;8:68863-72.

8. Churg A, Hwang H, Tan L, Qing G, Taher A, Tong A, et al. Malignant mesothelioma in situ. Histopathology. 2018;72:1033-8.

9. Berg KB, Dacic S, Miller C, Cheung S, Churg A. Utility of methylthioadenosine phosphorylase compared with BAP1 immunohistochemistry, and CDKN2A and NF2 fluorescence in situ hybridization in separating reactive mesothelial proliferations from epithelioid malignant mesotheliomas. Arch Pathol Lab Med. 2018;142:1549-53.

10. Chiosea S, Krasinskas A, Cagle PT, Mitchell KA, Zander DS, Dacic S. Diagnostic importance of 9p21 homozygous deletion in malignant mesotheliomas. Mod Pathol. 2008;21:742-57.

11. Pillappa R, Maleszewski JJ, Sukov WR, Bedroske PP, Greipp PT, Boland JM, et al. Loss of BAP1 expression in atypical mesothelial proliferations helps to predict malignant mesothelioma. Am J Surg Pathol. 2018;42:256-63.

12. Ostör AG. Natural history of cervical intraepithelial neoplasia: a critical review. Int J Gynecol Pathol 1993;12:186-92.

13. Krasinskas AM, Bartlett DL, Cieply K. Dacic S. CDKN2A and MTAP deletions in peritoneal mesotheliomas are correlated with loss of p16 protein expression and poor survival. Mod Pathol. 2010;23:531-48.

14. Hida T, Hamasaki M, Matsumoto S, Sato A, Tsujimura T, Kawahara K, et al. Immunohistochemical detection of MTAP and BAP1 protein loss for mesothelioma diagnosis: comparison with 9p21 FISH and BAP1 immunohistochemistry. Lung Cancer. 2017;104:98-105.

15. Chapel DB, Schulte JJ, Berg K, Churg A, Dacic S, Fitzpatrick C et al. MTAP immunohistochemistry is an accurate and reproducible surrogate for CDKN2A fluorescence in situ hybridization in diagnosis of malignant pleural mesothelioma. Mod Pathol. 2019. https://doi.org/10.1038/s41379-019-0310-0.

16. Bosio M, Salvaterra E, Datturi F, Morbini P, Zorzetto M, Inghilleri $\mathrm{S}$, et al. 5-hydroxymethylcytosine but not MTAP methylation status can stratify malignant pleural mesothelioma based on the lineage of origin. Multidiscip Respir Med. 2018;13:27.

17. Bueno R, Stawiski EW, Goldstein LD, Durinck S, De Rienzo A, Modrusan Z, et al. Comprehensive genomic analysis of malignant pleural mesothelioma identifies recurrent mutations, gene fusions and splicing alterations. Nat Genet. 2016;48:407-16.

18. Hmeljak J, Sanchez-Vega F, Hoadley KA, Shih J, Stewart C, Heiman D, et al. Integrative molecular characterization of malignant pleural mesothelioma. Cancer Discov. 2018;8:1548-65. 